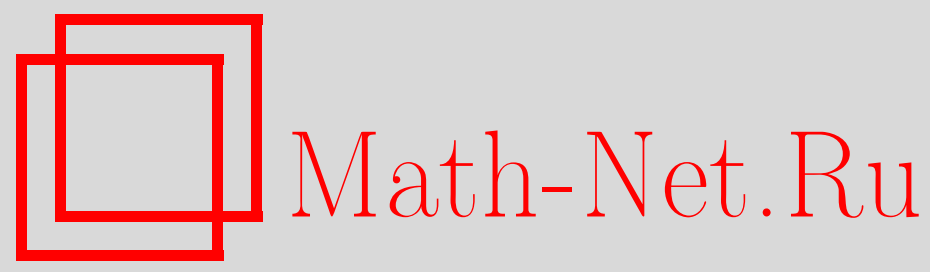

В. П. Деревенский, Системы матричных линейных дифференциальных уравнений первого порядка, Матем. заметкu, 1999, том 66, выпуск 1, 63-75

DOI: https://doi.org/10.4213/mzm1142

Использование Общероссийского математического портала Math-Net.Ru подразумевает, что вы прочитали и согласны с пользовательским соглашением http://www.mathnet.ru/rus/agreement

Параметры загрузки:

IP: 34.227 .88 .159

26 апреля 2023 г., $13: 28: 13$

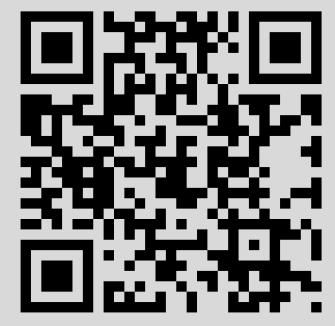




\section{СИСТЕМЫ МАТРИЧНЫХ ЛИНЕЙНЫХ ДИФФЕРЕНЦИАЛЬНЫХ УРАВНЕНИЙ ПЕРВОГО ПОРЯДКА}

\section{В. П. Деревенский}

В статье даются достаточные условия разрешимости в квадратурах систем матричных линейных обыкновенных дифференциальных уравнений первого порядка с односторонним умножением на переменные матричные коэффициенты. Эти условия формулируются в терминах теории алгебр Ли. Рассматриваются матричные уравнения высших порядков, эквивалентные таким системам. Имеется иллюстративный пример.

Библиография: 8 названий.

В статье определяются достаточные условия разрешимости в квадратурах системы матричных линейных обыкновенных дифференциальных уравнений первого порядка (СМЛОДУ.1) с левосторонним умножением

$$
\dot{X}_{i} \equiv \frac{d}{d t} X_{i}=A_{i}^{j} X_{j}+B_{i}, \quad i, j=1, \ldots, K, \quad A_{i}^{j}=A_{i}^{j}(t), \quad B_{i}=B_{i}(t),
$$

где $X_{i}$ - искомая, $A_{i}^{j}$ и $B_{i}$ - данные ограниченные по норме и непрерывные квадратные матрицы в собственно-евклидовом пространстве $\mathbb{R}_{n}$ над действительньм полем $\mathbb{R}$, а по повторяющимся верхним и нижним индексам производится суммирование.

1. Интегрирование системы матричных линейных обыкновенных дифференциальных уравнений первого порядка.. Задача о нахождении условий разрешимости в квадратурах систем (1) является естественным обобщением очень актуальных задач интегрирования матричных линейных дифференциальных уравнений первого порядка и систем скалярных ЛОДУ.1. Методы решения систем (1) позволяют, во-первых, упростить технику блочного оперирования при рассмотрении отдельных матричных линейных дифференциальных уравнений, а во-вторых, распространить классические методы интегрирования скалярных систем на некоммутативное множество. Такие задачи неизбежно появляются при рассмотрении первого линейного приближения динамики взаимодействия различных эволюционных процессов, описьваемых отдельными МЛОДУ.1. Особое значение подобных систем заключается в том, что они могут использоваться как представление систем линейно-операторных дифференциальных уравнений, которые всегда имеют матричное представление [1]. Так что излагаемые ниже методы и результаты практически полностью могут быть перенесены и на этот вид уравнений.

Обращаясь к системе (1), отметим, что она эквивалентна одному блочно-матричному уравнению, в котором искомая матрица и свободньй член имеют размерность $K n \times n$, 
а матричньй коэффищиент $-K n \times K n$. Но это уравнение может быть представлено как скалярная система $K n^{2}$ уравнений. Для нее справедлива теорема о существовании и единственности решений $[2$, с. 22$]$, дающая положительньй ответ и на аналогичный вопрос для систем (1).

При обобщении методов интегрирования скалярных систем на матричный случай необходимо дать способ решения систем матричных алгебраических линейных уравнений (СМАЛУ). Таким способом может быть "обобщенньй алгоритм Гаусса" [3, с. 55]. Формализуя его, дадим

ОПРЕДЕЛЕНИЕ 1. Левым оператором Гаусса блочной матрищы $A \equiv\left(A_{i}^{j}\right)$, у которой $A_{m}^{k}$ - невырожденная матрица, называется линейньй оператор $\Gamma\left(A_{m}^{k}\right)$, действующий на матричную последовательность $\left(Y_{l}\right)(l=1, \ldots, K)$ по правилу

$$
\Gamma\left(A_{m}^{k}\right) Y_{l} \equiv Y_{l}-A_{l}^{k}\left(A_{m}^{k}\right)^{-1} Y_{m}
$$

Так как $\Gamma\left(A_{m}^{k}\right) A_{l}^{k}=A_{l}^{k}-A_{l}^{k}\left(A_{m}^{k}\right)^{-1} A_{m}^{k} \equiv 0$, введенньй оператор реализует "обобщенный алгоритм Гаусса" решения СМАЛУ с левосторонним умножением. Повторное применение их задает

ОПРЕДЕЛЕНИЕ 2. Произведением операторов Гаусса назьвается

$$
\Gamma\left(A_{m}^{k}\right) \Gamma\left(B_{i}^{j}\right) Y_{l} \equiv \Gamma\left(\Gamma\left(B_{i}^{j}\right) A_{m}^{k}\right)\left(\Gamma\left(B_{i}^{j}\right) Y_{l}\right)
$$

В символах операторов Гаусса коэффищиенты алгебраической системы характеризует

ОПРЕДЕЛЕниЕ 3. Квадратно-блочная матрица $A$ назьвается невырожденной,если при некоторой перенумерации ее индексов матрица

$$
\Delta(A) \equiv A_{1}^{1} \prod_{i=2}^{K-1}\left(\prod_{k=1}^{i-1} \Gamma\left(A_{i-k}^{i-k}\right) A_{i}^{i}\right)
$$

неособенна, т.е.

$$
\delta(A) \equiv \operatorname{det} \Delta(A) \neq 0 .
$$

Теперь может быть сформулирована

Лемма 1. Решением системы матричных алгебраических линейных уравнений

$$
U_{i}^{j} X_{j}=V_{i}, \quad i, j=1, \ldots, N,
$$

с невырохденной матрицей $\left(U_{i}^{j}\right)$ является

$$
\begin{aligned}
X_{1} & =\left(U_{1}^{1}\right)^{-1} \prod_{\tau=N}^{2} \Gamma\left(\widetilde{U}_{\tau}^{\tau}\right) V_{1}, \quad \tilde{U}_{\tau}^{\tau}=\prod_{k=1}^{\tau-1} \Gamma\left(U_{\tau-k}^{\tau-k}\right) U_{\tau}^{\tau}, \\
X_{i} & =\left(\prod_{k=1}^{i-1} \Gamma\left(U_{i-k}^{i-k}\right) U_{i}^{i}\right)^{-1} \prod_{\tau=N}^{i+1} \Gamma\left(\widetilde{U}_{\tau}^{\tau}\right)\left(\prod_{k=1}^{i-1} \Gamma\left(U_{i-k}^{i-k}\right) V_{i}\right), \\
X_{N} & =\left(\prod_{k=1}^{N-1} \Gamma\left(U_{N-k}^{N-k}\right) U_{N}^{N}\right)^{-1} \prod_{k=1}^{N-1} \Gamma\left(U_{N-k}^{N-k}\right) V_{N} .
\end{aligned}
$$


ДокАЗАтЕльСтво. Оператор $\Gamma\left(U_{1}^{1}\right)$ позволяет обратить в нуль коэффициенты при $X_{1}$ во всех уравнениях, начиная со второго. Получаемая при этом подсистема $(N-1)$-го порядка, $\Gamma\left(U_{1}^{1}\right) U_{i}^{j} X_{j}=\Gamma\left(U_{1}^{1}\right) V_{i}$, в силу невырожденности матрицы коэффищиентов допускает такую перенумерацию индексов, при которой коэффициент у $X_{2}$ во втором уравнении будет невырожденным. Это позволяет действием оператора $\Gamma\left(\Gamma\left(U_{1}^{1}\right) U_{2}^{2}\right)$ на третье и последующие уравнения получить нулевые коэффициенты при $X_{2}$. Однако согласно (2) $\Gamma\left(\Gamma\left(U_{1}^{1}\right) U_{2}^{2}\right)\left(\Gamma\left(U_{1}^{1}\right) U_{1}^{j}\right) \equiv \Gamma\left(U_{2}^{2}\right) \Gamma\left(U_{1}^{1}\right) U_{1}^{j}$. Следовательно, при продолжении этого процесса производится “прямой ход” [4, с. 157] матричного метода Гаусса, триангулируюшего систему (5),

$$
\begin{gathered}
U_{1}^{j} X_{j}=V_{i}, \quad j=1, \ldots, N, \\
\Gamma\left(U_{1}^{1}\right) U_{2}^{j} X_{j}=\Gamma\left(U_{1}^{1}\right) V_{2}, \quad j=2, \ldots, N, \\
\ldots \ldots \ldots \ldots \ldots \ldots \ldots \ldots \ldots \ldots \ldots \ldots \ldots \ldots \ldots \\
\prod_{k=1}^{N-1} \Gamma\left(U_{N-k}^{N-k}\right) U_{N}^{N} X_{N}=\prod_{k=1}^{N-1} \Gamma\left(U_{N-k}^{N-k}\right) V_{N} .
\end{gathered}
$$

“Обратный ход” метода Гаусса, диагонализирующий полученную треугольную систему, осуществляется действиями операторов $\Gamma\left(U_{i-k}^{i-k}\right)$ на уравнения в обратном порядке с помощью преобразованной “прямым ходом” матрицы коэффициентов. Для наглядности операторы "обратного хода" нумеруются греческими индексами. При этом требование (3) гарантирует и существование операторов Гаусса, и разрешимость диагонализованной системы, так как “обратньй ход” не меняет диагональных коэффициентов системы, которая принимает вид

$$
\prod_{k=1}^{i-1} \Gamma\left(U_{i-k}^{i-k}\right) U_{i}^{i} X_{i}=\prod_{\tau=N}^{i+1} \Gamma\left(\tilde{U}_{\tau}^{\tau}\right)\left(\prod_{k=1}^{i-1} \Gamma\left(U_{i-k}^{i-k}\right) V_{i}\right)
$$

Откуда и следует (6).

Заметим, что найти $X_{i}$ можно простым переносом внедиагональных слагаемых триангулированной системы за знак равенства с последуюшим умножением на

$$
\left(\prod_{k=1}^{i-1} \Gamma\left(U_{i-k}^{i-k}\right) U_{i}^{i}\right)^{-1}
$$

При этом не используются операторы “обратного хода", а $X_{i}$ записываются в виде, соответствуюшем традиционному способу решения скалярных систем:

$$
X_{i}=\left(\prod_{k=1}^{i-1} \Gamma\left(U_{i-k}^{i-k}\right) U_{i}^{i}\right)^{-1} \prod_{k=1}^{i-1} \Gamma\left(U_{i-k}^{i-k}\right)\left(V_{i}-A_{i}^{j} X_{j}\right)
$$

Теперь можно дать условия разрешимости СМЛОДУ.1. 
Лемма 2. Однородная система (1) разрешима в квадратурах, если $A_{i}^{j}=0$ : $(i>j) \vee(i<j)$, а $A_{i}^{i}$ принадлежсат представлениям в $\mathbb{R}_{n}$ разрешимых $N(i)$-мерных алгебр Ли $L_{N(i)}$.

ДокАЗАТЕЛЬСтво. Для первого дизъюнктного условия последнее уравнение в однородной системе (1) принимает вид $\dot{X}_{K}=A_{K}^{K} X_{K}, A_{K}^{K} \in L_{N(K)}$. В этом случае [5] существует $X_{K}=\exp \left(b_{K}^{\alpha} E_{K \alpha}\right)(\alpha=1, \ldots, N(K))$, где $b_{K}^{\alpha}$ - скалярные функции класса $C^{1}(t)$, а $E_{K \alpha}$ - базисные матрицы $L_{N(K)}$. В силу ее разрешимости $b_{K}^{\alpha}$ определяются в квадратурах. Для $i<K$

$$
\dot{X}_{i}=A_{i}^{i} X_{i}+\sum_{r=1}^{K-i} A_{i}^{i+r} X_{i+r}
$$

Следовательно,

$$
X_{i}=X_{i 0} \int X_{i 0}^{-1} \sum_{r=1}^{K-i} A_{i}^{i+r} X_{i+r} d t
$$

где $X_{i 0}$ - невырожденные решения однородных уравнений $\dot{X}_{i 0}=A_{i}^{i} X_{i 0}$, для которых при принадлежности $A_{i}^{i}$ разрешимьм $L_{N(i)}$ экспоненциальные решения определяются в квадратурах. Последовательное нахождение $X_{i}$ через $X_{i+r}$ обеспечивает разрешимость в квадратурах всей рассматриваемой системы. Для второго дизъюнктного условия рассуждения повторяются в порядке возрастания индекса $i$. Заметим, что интегрируемость МЛОДУ.1 в альтернативном мультипликативно-экспоненциальном виде для разрешимых алгебр Ли, содержащих матричньй параметр уравнения, доказана в [6].

Как и в скалярном случае, свободные члены системы (1) не имеют существенного значения, так как "метод вариации постоянных" обобщается и на СМЛОДУ.1. Прежде чем обосновать это, отметим, что при сохранении классического определения фундаментальной системы решений системы (1) необходимым и достаточным условием линейной независимости $K$ ее решений, нумеруемых верхним индексом, $X_{i}^{j}$, будет (4), т.е. $\delta(X) \neq 0$.

Лемма 3. Решением системы (1) является

$$
\begin{aligned}
& X_{i}=X_{i 0}^{j} Y_{j}, \quad \dot{X}_{i 0}^{j}=A_{i}^{k} X_{k 0}^{j}, \quad \delta\left(X_{0}\right) \neq 0 \\
& Y_{i}=\int\left(\prod_{k=1}^{i-1} \Gamma\left(X_{i-k, 0}^{i-k}\right) X_{i 0}^{i}\right)^{-1} \prod_{\tau=K}^{i+1} \Gamma\left(\tilde{X}_{\tau 0}^{\tau}\right) \prod_{k=1}^{i-1} \Gamma\left(X_{i-k, 0}^{i-k}\right) B_{i} d t
\end{aligned}
$$

ДокАЗАТЕЛЬСтво. Если подставить в (1) $X_{i}=X_{i 0}^{j} Y_{j}$, то на $Y_{j}$ наложатся условия $X_{i 0}^{j} \dot{Y}_{j}=B_{i}$. Эта СМАЛУ разрешима относительно $\dot{Y}_{j}$, так как блочная матрища ее коэффициентов невырождена по условию. Выбирая нумерацию индексов фундаментальной системы решений однородной системы так, чтобы матрица $\Delta\left(X_{0}\right)$ имела вид $(3)$, определим $\dot{Y}_{j}$ в форме подинтегральных функций в $(8)$, что и требовалось показать.

Из приведенных утверждений следует 
ТЕОрема 1. Если в системе (1) перенумерацией индексов $A_{i}^{j}$ можнно представить в виде

$$
A_{i}^{j}=\left(\prod_{k=1}^{i-1} \Gamma\left(T_{i-k}^{i-k}\right)\left(F_{i}^{m} T_{m}^{i}\right)\right)^{-1} \prod_{\tau=K}^{i+1} \Gamma\left(\widetilde{T}_{\tau}^{\tau}\right) \prod_{k=1}^{i-1} \Gamma\left(T_{i-k}^{i-k}\right)\left(F_{i}^{m} T_{m}^{j}\right),
$$

əəe $\left\|F_{i}^{j}\right\|<\infty$,

$$
F_{i}^{j}= \begin{cases}0, & i<j, \\ f_{i}^{\alpha} E_{i \alpha}, & E_{i \alpha} \in L_{N(i)}, f_{i}^{\alpha} \in C^{1}(t), i=j, \\ F_{i}^{j}, & i>j,\end{cases}
$$

а $L_{N(i)}$ - разрешима, то она интегрируется в квадратурах.

ДокАЗАТЕльСтво. Как следует из (6), условие (9) определяет блочную матрицу $\left(A_{i}^{j}\right)$ как решение системы $T_{i}^{j} A_{j}^{k}=F_{i}^{j} T_{j}^{k}$. Следовательно, свертывая (1) с $T_{k}^{i}$, эту систему можно записать в виде $\dot{Z}_{i}=F_{i}^{j} Z_{j}+Q_{i}, Z_{i}=T_{i}^{k} X_{k}, Q_{i}=T_{i}^{k} B_{k}$. В силу леммы 3 решение этой системы сводится к решению однородной, а она по условию (10) является треугольной. Разрешимость $L_{N(i)}$, содержаших диагональные элементы $\left(F_{i}^{j}\right)$, обеспечивает в соответствии с леммой 2 интегрируемость в квадратурах однородных систем $\dot{Z}_{i 0}=F_{i}^{i} Z_{i 0}$. Существование же фундаментальной системы решений однородной СМЛОДУ.1 доказывается аналогично скалярному случаю [2, c. 130].

ЗАмЕчАниЕ. Все сказанное вьше справедливо и для правосторонних систем $\dot{X}_{i}=$ $X_{j} A_{i}^{j}+B_{i}$ при коммутации сомножителей в произведениях.

Работая в двумерном пространстве, полезно учитьвать следующее

ПРЕДЛОЖЕНИЕ. $В \mathbb{R}_{2}$ наиболее общим видом систем, удовлетворяющих условиям теоремы 1, являются (1) с $A_{i}^{j}$ вида (9), әде

$$
F_{i}^{i}=\left(\begin{array}{cc}
\varphi_{i 1} & \varphi_{i 2} \\
\varphi_{i 3} & \varphi_{i 1}+\alpha_{i} \varphi_{i 2}-\alpha_{i}^{-1} \varphi_{i 3}
\end{array}\right), \quad \dot{\alpha}_{i}=0, \quad \varphi_{i r} \in C^{1}(t), \quad r=1,2,3,
$$

а решением ее будет

$$
\begin{aligned}
X_{i} & =\left(\prod_{k=1}^{i-1} \Gamma\left(T_{i-k}^{i-k}\right) T_{i}^{i}\right)^{-1} \prod_{\tau=K}^{i+1} \Gamma\left(\widetilde{T}_{\tau}^{\tau}\right) \prod_{k=1}^{i-1} \Gamma\left(T_{i-k}^{i-k}\right) Z_{i}, \\
Z_{i} & =Z_{i 0} \int Z_{i 0}^{-1}\left(F_{i}^{k} Z_{k}+T_{i}^{j} B_{j}\right) d t, \quad k \neq i,
\end{aligned}
$$

$2 \partial e$

$$
\begin{gathered}
Z_{i 0}=a_{i}\left(\begin{array}{cc}
\operatorname{ch} \tau_{i}-b_{i} & \alpha_{i}^{-1}\left(\operatorname{sh} \tau_{i}+b_{i}\right) \\
-\alpha_{i}\left(\operatorname{sh} \tau_{i}+b_{i}\right) & \operatorname{ch} \tau_{i}+b_{i}
\end{array}\right) \\
a_{i}=\exp \frac{1}{2} \int_{0}^{t}\left(2 \varphi_{i 1}+\alpha_{i} \varphi_{i 2}-\alpha_{i}^{-1} \varphi_{i 3}\right) d t, \quad b_{i}=\frac{1}{2} \int_{0}^{t}\left(\alpha_{i} \varphi_{i 2}-\alpha_{i}^{-1} \varphi_{i 3}\right) \exp \left(2 \tau_{i}\right) d t \\
\tau_{i}=\frac{1}{2} \int_{0}^{t}\left(\alpha_{i} \varphi_{i 2}+\alpha_{i}^{-1} \varphi_{i 3}\right) d t
\end{gathered}
$$


ДокАЗАТЕльСТво. В (11) дан наиболее общий вид матрицы, принадлежащей разрешимой $L_{3}[7]$, являюшейся прямой суммой двумерной разрешимой алгебры и одномерного центра. Если за базис ее взять матрицы

$$
E_{i 1}=\left(\begin{array}{cc}
-\alpha_{i} & 1 \\
-\alpha_{i}^{-1} & \alpha_{i}
\end{array}\right), \quad E_{i 2}=-\frac{1}{2}\left(\begin{array}{cc}
0 & \alpha_{i}^{-1} \\
\alpha_{i} & 0
\end{array}\right), \quad E_{i 3}=E,
$$

то $F_{i}^{i}=f_{i}^{\alpha} E_{i \alpha}$, где $f_{i}^{1}=\left(\varphi_{i 2}-\alpha_{i}^{-1} \varphi_{i 3}\right) / 2, f_{i}^{2}=-\alpha_{i} \varphi_{i 2}-\alpha_{i}^{-1} \varphi_{i 3}, f_{i}^{3}=\varphi_{i 1}+f_{i}^{1}$, причем $\left[E_{i 1} E_{i 2}\right]=E_{i 1},\left[E_{i \alpha} E_{i 3}\right]=0(\alpha=1,2,3)$. Следовательно, если в системе (1) перейти к неизвестным $Z_{k}=T_{k}^{i} X_{i}$, то она становится треугольной, $\dot{Z}_{i}=F_{i}^{j} Z_{j}+Q_{i}, F_{i}^{j}=$ $T_{i}^{k} A_{k}^{l}\left(T^{-1}\right)_{l}^{j}, Q_{i}=T_{i}^{j} B_{j}$. При этом $X_{i}$ находятся через $Z_{k}$ по формулам $(6)$ в виде $(12)$, а метод вариации постоянных позволяет найти $Z_{k}$ в виде (13), где альтернативные варианты треугольности объединены способом суммирования по $k$. Таким образом, задача интегрирования системы (1) сводится к решению $K$ уравнений $\dot{Z}_{i 0}=f_{i}^{\alpha} E_{i \alpha} Z_{i 0}$. Определяя $Z_{i 0}$ в мультипликативно-экспоненциальном виде [5], [6] $Z_{i 0}=\prod_{\alpha=1}^{3} \exp \left(g_{i \alpha} E_{i \alpha}\right)$ $\left(g_{i \alpha} \in C^{1}(t), g_{i \alpha}(0)=0\right)$, найдем $g_{i \alpha}$ из условий $\dot{g}_{i 1}+\dot{g}_{i 2} g_{i 1}=f_{i}^{1}, \dot{g}_{i s}=f_{i}^{s}(s=2,3)$, что дает

$$
g_{i 1}=\exp \int_{t}^{0} f_{i}^{2} d t \int_{0}^{t} f_{i}^{1} \exp \int_{0}^{t} f_{i}^{2} d t d t \quad \text { и } \quad g_{i s}=\int_{0}^{t} f_{i}^{s} d t .
$$

Taк как

$$
\exp \left(g_{i 3} E\right)=E \exp g_{i 3},
$$

$\exp \left(g_{i 1} E_{i 1}\right)=\left(\begin{array}{cc}1-\alpha_{i} g_{i 1} & g_{i 1} \\ -\alpha_{i}^{2} g_{i 1} & 1+\alpha_{i} g_{i 1}\end{array}\right), \quad \exp \left(g_{i 2} E_{i 2}\right)=\left(\begin{array}{cc}\operatorname{ch} \frac{1}{2} g_{i 2} & -\alpha_{i}^{-1} \operatorname{sh} \frac{1}{2} g_{i 2} \\ -\alpha_{i} \operatorname{sh} \frac{1}{2} g_{i 2} & \operatorname{ch~} \frac{1}{2} g_{i 2}\end{array}\right)$,

перемножая эти экспоненты и используя введенные обозначения для $a_{i}, b_{i}$ и $\tau_{i}$, получаем (14), что и требовалось показать.

Чтобы сделать теорему 1 более наглядной и конкретной, приведем

СлЕДСТВИЕ. Если в двумерной системе (1) можно так перенумеровать индексы, что

$$
\begin{gathered}
T_{1}^{i} A_{i}^{1}\left(T_{1}^{1}\right)^{-1} T_{1}^{2}=T_{1}^{i} A_{i}^{2}, \quad A_{1}^{i}=\left(T_{1}^{1}\right)^{-1}\left(P_{1} T_{1}^{i}-T_{1}^{2} A_{2}^{i}\right) \\
A_{2}^{i}=H^{-1}(T)\left(P_{3} T_{1}^{i}+P_{2} T_{2}^{i}-T_{2}^{1}\left(T_{1}^{1}\right)^{-1} P_{1} T_{1}^{i}\right)
\end{gathered}
$$

әде $T_{i}^{j}$ - постоянные матрицы, $T=\left(T_{i}^{j}\right), H(T)=T_{2}^{2}-T_{2}^{1}\left(T_{1}^{1}\right)^{-1} T_{1}^{2}, \delta(T) \neq 0$, $P_{i}=p_{i}^{\alpha} E_{i \alpha},\left[E_{i \alpha} E_{i \beta}\right]=C_{\alpha \beta}^{\gamma}(i) E_{i \gamma}, E_{i \alpha} \in L_{N(i)}$, әлементы $P_{3}$ и $p_{i}^{\alpha}$ непрерывны, а $L_{N(i)}$ разрешимы, то решение СМЛОДУ.1 будет находиться в квадратурах в виде

$$
\begin{aligned}
X_{i} & =X_{i 0}^{j} Y_{j}, \quad \delta\left(X_{0}\right) \neq 0, \quad X_{0} \equiv\left(X_{i 0}^{j}\right) \\
Y_{1} & =\int\left(X_{10}^{1}\right)^{-1}\left(\left(E+X_{10}^{2} H^{-1}\left(X_{0}\right) X_{20}^{1}\left(X_{10}^{1}\right)^{-1}\right) B_{1}-X_{10}^{2} H^{-1}\left(X_{0}\right) B_{2}\right) d t \\
Y_{2} & =\int H^{-1}\left(X_{0}\right)\left(B_{2}-X_{20}^{1}\left(X_{10}^{1}\right)^{-1} B_{1}\right) d t \\
X_{10}^{j} & =\left(T_{1}^{1}\right)^{-1}\left(\left(E+T_{1}^{2} H^{-1}(T) T_{2}^{1}\left(T_{1}^{1}\right)^{-1} U_{1} Q^{j}-T_{1}^{2} H^{-1}(T) V^{j}\right),\right. \\
X_{20}^{j} & =H^{-1}(T)\left(V^{j}-T_{2}^{1}\left(T_{1}^{1}\right)^{-1} U_{1} Q^{j}\right),
\end{aligned}
$$


əде $V^{j}=U_{2} \int U_{2}^{-1} P_{3} U_{1} Q^{j} d t, Q^{j}-$ постоянные матриць,

$$
U_{i}=\exp \left(u_{i}^{\alpha} E_{i \alpha}\right), \quad \dot{u}_{i}^{\alpha}=p_{i}^{\gamma}\left(\sum_{n=0}^{\infty} \frac{1}{(n+1) !}\left(u_{i}^{\beta} C_{\alpha \beta}^{\gamma}(i)\right)^{n}\right)^{-1}, \quad u_{i}^{\alpha}(0)=0
$$

ДокАЗАТЕЛЬСТВО. Производя в двумерной системе (1) такую перенумерацию индексов, при которой $A_{i}^{j}$ удовлетворяют условию (4), определим $X_{i}=X_{i 0}^{j} Y_{j}$. Тогда на $Y_{j}$ наложатся условия $X_{i 0}^{j} \dot{Y}_{j}=B_{i}$. Одноэтапньй “прямой ход” метода Гаусса осушествляет преобразование второго уравнения оператором $\Gamma\left(X_{10}^{1}\right)$ к виду $\Gamma\left(X_{10}^{1}\right) X_{20}^{2} \dot{Y}_{2}=$ $\Gamma\left(X_{10}^{1}\right) B_{2}$. Это равенство можно расписать как

$$
\left(X_{20}^{2}-X_{20}^{1}\left(X_{10}^{1}\right)^{-1} X_{10}^{2}\right) \dot{Y}_{2}=B_{2}-X_{20}^{1}\left(X_{10}^{1}\right)^{-1} B_{1}
$$

что в силу невырожденности $X_{0}$ позволяет проделать “обратный ход” с помощью преобразованной матрицы $X_{20}^{2}, H\left(X_{0}\right)=\Gamma\left(X_{10}^{1}\right) X_{20}^{2}$. При этом изменяется лишш свободный член первого уравнения, принимающего вид $X_{10}^{1} \dot{Y}_{1}=B_{1}-X_{10}^{2} H^{-1}\left(X_{0}\right)\left(\Gamma\left(X_{10}^{1}\right) B_{2}\right)$. Так что требование невырожденности $X_{0}$ обеспечивает возможность найти $\dot{Y}_{j}$ из диагонализованной системы и проинтегрировать их в указанном виде. Кстати, тот же результат дает и формула Фробениуса [3, с. 60], определяющая матрицу, обратную к блочной $(2 n \times 2 n)$-матрище.

Найдем $X_{i 0}^{j}$, для чего решим однородную СМЛОДУ. 1 , вводя новые неизвестные $U_{k}^{j}=$ $T_{k}^{i} X_{i 0}^{j}$, где $T_{k}^{i}$ - постоянные матрищы с $\delta(T) \neq 0$. Первое из трех наложенных на $A_{i}^{j}$ условий означает, что матрица $P \equiv\left(P_{i}^{j}\right)$ в определяющем ее равенстве $T A=P T$ является нижнетреугольной, причем $P_{i}^{i} \equiv P_{i}$, а $P_{2}^{1} \equiv P_{3}$. Второе и третье условия определяют решения этого матричного равенства относительно $A_{k}^{i}$. Действительно, расписьвая его по компонентам, имеем

$$
T_{1}^{1} A_{1}^{i}+T_{1}^{2} A_{2}^{i}=P_{1} T_{1}^{i}, \quad T_{2}^{1} A_{1}^{i}+T_{2}^{2} A_{2}^{i}=P_{3} T_{1}^{i}+P_{2} T_{2}^{i}
$$

Откуда $\Gamma\left(T_{1}^{1}\right) T_{2}^{2} A_{2}^{i}=\left(T_{2}^{2}-T_{2}^{1}\left(T_{1}^{1}\right)^{-1} T_{1}^{2}\right) A_{2}^{i}=P_{3} T_{1}^{i}+P_{2} T_{2}^{i}-T_{2}^{1}\left(T_{1}^{1}\right)^{-1} P_{1} T_{1}^{i}$. Матрица $A_{1}^{i}$ дана в виде (7), однако ее можно представить в эквивалентной форме (6), так как

$$
T_{1}^{1} A_{1}^{i}=\Gamma\left(T_{2}^{2}\right) \Gamma\left(T_{1}^{1}\right) P_{1} T_{1}^{i}=P_{1} T_{1}^{i}-T_{1}^{2}\left(\Gamma\left(T_{1}^{1}\right) T_{2}^{2}\right)^{-1} \Gamma\left(T_{1}^{1}\right)\left(P_{3} T_{1}^{i}+P_{2} T_{2}^{i}\right)=P_{1} T_{1}^{i}-T_{1}^{2} A_{2}^{i}
$$

Таким образом, мы имеем матричную систему: $\dot{Z}_{1}^{i}=P_{1} Z_{1}^{i}, \dot{Z}_{2}^{i}=P_{2} Z_{2}^{i}+P_{3} Z_{1}^{i}$. Решением первой пары этих уравнений будет $Z_{1}^{i}=U_{1} Q_{1}^{i}$, где $U_{1}$ - нормированное решение, а $Q_{1}^{i}$ - постоянные матрицы. При этом $U_{1}$ находится в экспоненциальной форме, показатель которой имеет проекции на базис $L_{N(1)}, u_{1}^{\alpha}$, удовлетворяющие данной скалярной нелинейной системе [5]. Решение второй пары матричных уравнений имеет вид [2, с. 135] $Z_{2}^{i}=Z_{20}^{i} \int\left(Z_{20}^{i}\right)^{-1} P_{3} Z_{1}^{i} d t$, в котором $Z_{20}^{i}$ - невырожденные решения соответствующих однородньх уравнений. Таким образом, $Z_{2}^{i}=U_{2}\left(R Q_{1}^{i}+Q_{2}^{i}\right)$, где $U_{2}-$ нормированное решение однородного МЛОДУ.1, также имеющее вид $U_{2}=\exp \left(u_{2}^{\alpha} E_{2 \alpha}\right)$, $R=\int U_{2}^{-1} P_{3} U_{1} d t$, а $Q_{2}^{i}$-постоянные матрицы. Легко убедиться, что $H(Z)=U_{2} H(Q)$, т.е. $(\delta(X) \neq 0) \Longleftrightarrow(\delta(Z) \neq 0) \Longleftrightarrow(\delta(Q) \neq 0)$. 
2. Уравнения $K$-го порядка. Формулы (6) позволяют определить матричные линейные обыкновенные дифференциальные уравнения $K$-го порядка (МЛОДУ.К), эквивалентные СМЛОДУ.1. Во избежание громоздких записей будем рассматривать однородные уравнения. Это не ограничивает их общности, так как и в матричном случае действует "метод вариации постоянных" , который формулируется следующим образом.

ЛЕмма 4. Общим решением матричного уравнения

$$
\sum_{i=0}^{K} A_{i} X^{(K-i)}=F
$$

с непрерывными матрицами $A_{i}$ и F является

$$
\begin{gathered}
X=X_{j 0} T^{j}, \quad \sum_{i=0}^{K} A_{i} X_{j 0}^{(K-i)}=0, \quad \delta\left(X_{j 0}^{(l)}\right) \neq 0, \\
j=1, \ldots, K, \quad l=0, \ldots, K-1, \quad\left(F_{l}\right) \equiv(0, \ldots, 0, F), \\
T^{j}=\int\left(\prod_{k=1}^{j-1} \Gamma\left(X_{j-k, 0}^{(j-k-1)}\right) X_{j 0}^{(j-1)}\right)^{-1} \prod_{\tau=K}^{j+1} \Gamma\left(\tilde{X}_{\tau}^{(\tau-1)}\right) F_{K-1} d t .
\end{gathered}
$$

ДокАЗАтЕльство. Так как в уравнении (15) используются левосторонние произведения, классический метод вариации постоянных [2, с. 143] необходимо обусловить лишь правосторонним умножением решений однородного уравнения на матричные константы. А условия на эти матрищы, как и наличие фундаментальной системы однородного уравнения, устанавливаются обычным способом. Решение же матричной системы

$$
\left\{\begin{array}{l}
X_{j 0}^{(\alpha)} \dot{T}^{j}=0, \quad \alpha=0, \ldots, K-2, \\
X_{j 0}^{(K-1)} \dot{T}^{j}=F
\end{array}\right.
$$

относительно $\dot{T}^{j}$ определяется по формулам (6) в виде подинтегральных функций в (16). При этом, так как "прямой ход" метода Гаусса в силу однородности первых $K-1$ уравнений не меняет $F$, то в $T^{j}$ матрицу $F$ преобразуют лиш операторы Гаусса "обратного хода".

Таким образом, при рассмотрении МЛОДУ.К можно ограничиться однородньми уравнениями, порождаемьми системой

$$
\dot{X}_{i}=A_{i}^{j} X_{j}, \quad\left\|A_{i}^{j}\right\|<\infty, \quad i, j=1, \ldots, K
$$

в которой элементы матриц $A_{i}^{j}$ принадлежат $C^{k}(t)$. Как и в скалярном случае, система (17) порождает $K$ матричных однородных уравнений $K$-го порядка. Для них условия разрешимости в квадратурах дает теорема 1. Чтобы воспользоваться ими, введем обозначения

$$
X_{i}^{(l)}=A_{i l}^{j} X_{j}, \quad A_{i l}^{j} \equiv \dot{A}_{i, l-1}^{j}+A_{i, l-1}^{k} A_{k}^{j}, \quad A_{i 1}^{j} \equiv A_{i}^{j} .
$$


Совокупность $K-1$ этих равенств образует СМАЛУ относительно $X_{\alpha}(i \neq \alpha=$ $1, \ldots, K) A_{i l}^{\alpha} X_{\alpha}=X_{i}^{(l)}-A_{i l}^{i} X_{i}, l=1, \ldots, K-1$, решение которой при $i \neq 1$ (наиболее обший случай) согласно (6) имеет вид

$$
\begin{aligned}
X_{\alpha} & =\left(\prod_{k=1}^{\alpha-1} \Gamma\left(A_{i, \alpha-k}^{\alpha-k}\right) A_{i \alpha}^{\alpha}\right)^{-1} \prod_{\tau=K-1}^{\alpha+1} \Gamma\left(\widetilde{A}_{i \tau}^{\tau}\right) \prod_{k=1}^{\alpha-1} \Gamma\left(A_{i, \alpha-k}^{\alpha-k}\right)\left(X_{i}^{(\alpha)}-A_{i \alpha}^{i} X_{i}\right), \\
X_{\beta} & =\left(\prod_{k=2}^{\beta-1} \Gamma\left(A_{i, \beta-k}^{\beta-k}\right) A_{i, \beta-1}^{\beta}\right)^{-1} \prod_{\tau=K-1}^{\beta+1} \Gamma\left(\widetilde{A}_{i \tau}^{\tau}\right) \prod_{k=2}^{\beta-1} \Gamma\left(A_{i, \beta-k}^{\beta-k}\right)\left(X_{i}^{(\beta-1)}-A_{i, \beta-1}^{i} X_{i}\right), \\
\alpha & <i<\beta
\end{aligned}
$$

(решение записано для такой нумерации индексов, при которой $\delta\left(A_{i l}^{\alpha}\right) \neq 0$ ). Если же $i=1$, то

$$
X_{\alpha}=\left(\prod_{k=2}^{\alpha-1} \Gamma\left(A_{i, \alpha-k}^{\alpha-k}\right) A_{1, \alpha-1}^{\alpha}\right)^{-1} \prod_{\tau=K-1}^{\alpha} \Gamma\left(\widetilde{A}_{1 \tau}^{\tau}\right) \prod_{k=2}^{\alpha-1} \Gamma\left(A_{1, \alpha-k}^{\alpha-k}\right)\left(X_{1}^{(\alpha-1)}-A_{1, \alpha-1}^{1} X_{1}\right) .
$$

Подставляя $X_{\alpha}$ из (19) в равенство $X_{i}^{(K)}=A_{i K}^{\alpha} X_{\alpha}+A_{i}^{i} X_{i}$, где по $i$ нет суммирования, получаем все $K$ МЛОДУ.К:

$$
\begin{aligned}
X_{i}^{(K)}= & A_{i K}^{1}\left(A_{i}^{i}\right)^{-1} \prod_{\tau=K-1}^{2} \Gamma\left(\tilde{A}_{i \tau}^{\tau}\right)\left(\dot{X}_{i}-A_{i}^{i} X_{i}\right) \\
& +\sum_{\alpha=2}^{i-1} A_{i K}^{\alpha}\left(\prod_{k=1}^{\alpha-1} \Gamma\left(A_{i, \alpha-k}^{\alpha-k}\right) A_{i \alpha}^{\alpha}\right)^{-1} \\
& \times \prod_{\tau=K-1}^{\alpha+1} \Gamma\left(\widetilde{A}_{i \tau}^{\tau}\right) \prod_{k=1}^{\alpha-1} \Gamma\left(A_{i, \alpha-k}^{\alpha-k}\right)\left(X_{i}^{(\alpha)}-A_{i \alpha}^{i} X_{i}\right) \\
& +\sum_{\beta=i+1}^{K-1} A_{i K}^{\beta}\left(\prod_{k=2}^{\beta-1} \Gamma\left(A_{i, \beta-k}^{\beta-k}\right) A_{i, \beta-1}^{\beta}\right)^{-1} \\
& \times \prod_{\tau=K-1}^{\beta+1} \Gamma\left(\tilde{A}_{i \tau}^{\tau}\right) \prod_{k=2}^{\beta-1} \Gamma\left(A_{i, \beta-k}^{\beta-k}\right)\left(X_{i}^{(\beta-1)}-A_{i, \beta-1}^{i} X_{i}\right) \\
& +A_{i K}^{K}\left(\prod_{k=2}^{K-1} \Gamma\left(A_{i, K-k}^{K-k}\right) A_{i, K-1}^{K}\right)^{-1} \prod_{k=2}^{K-1} \Gamma\left(A_{i, K-k}^{K-k}\right)\left(X_{i}^{(K-1)}-A_{i, K-1}^{i} X_{i}\right)
\end{aligned}
$$

для которых справедлива

ТЕорема 2. Если в уравнении (20) $A_{i l}^{j}$ определяются в виде (18), а $A_{i}^{j}$ при перенумерации $i$ и мохнно представить в виде (9), то оно интегрируется $\boldsymbol{в}$ квадратурах.

Доказательство этого утверждения следует из эквивалентности решений, полученных МЛОДУ.К и исходной СМЛОДУ.1, которая при условии (9) интегрируется в квадратурах в силу теоремы 1 . 
3. ПримеР. Пусть в системе (1) $K=2$. Тогда

$$
\frac{d}{d t}\left(\begin{array}{l}
X_{1} \\
X_{2}
\end{array}\right)=\left(\begin{array}{cc}
\left(\begin{array}{cc}
0 & -t \\
\frac{1}{2}(1-t) & 0
\end{array}\right) & \left(\begin{array}{cc}
0 & -t \\
\frac{1}{2} t & -1-2 t
\end{array}\right) \\
\left(\begin{array}{cc}
1 & t \\
-\frac{1}{2}(1+t) & 0
\end{array}\right) & \left(\begin{array}{cc}
1 & t \\
-\frac{1}{2} t & 1+2 t
\end{array}\right)
\end{array}\right)\left(\begin{array}{l}
X_{1} \\
X_{2}
\end{array}\right)+\left(\begin{array}{c}
\left(\begin{array}{cc}
0 & -1 \\
0 & 0
\end{array}\right) \\
\left(\begin{array}{ll}
0 & 1 \\
0 & 0
\end{array}\right)
\end{array}\right)
$$

Эта система триангулируется преобразованием $T_{i}^{j} A_{j}^{k}=F_{i}^{j} T_{j}^{k}$, где

$$
\left(T_{i}^{j}\right)=\left(\begin{array}{cc}
\left(\begin{array}{cc}
0 & 1 \\
-1 & 0
\end{array}\right) & \left(\begin{array}{ll}
0 & 1 \\
0 & 0
\end{array}\right) \\
\left(\begin{array}{ll}
1 & 0 \\
0 & 0
\end{array}\right) & \left(\begin{array}{ll}
1 & 0 \\
0 & 2
\end{array}\right)
\end{array}\right), \quad\left(F_{i}^{j}\right)=\left(\begin{array}{ll}
\left(\begin{array}{ll}
0 & t \\
t & 0
\end{array}\right) & \left(\begin{array}{ll}
0 & 0 \\
0 & 0
\end{array}\right) \\
\left(\begin{array}{ll}
0 & 0 \\
0 & 1
\end{array}\right) & \left(\begin{array}{cc}
1 & t \\
-t & 1+2 t
\end{array}\right)
\end{array}\right) .
$$

Следовательно, система (21) может быть записана в виде

$$
\left\{\begin{array}{l}
\dot{Z}_{1}=F_{1}^{1} Z_{1}+Q_{1}, \\
\dot{Z}_{2}=F_{2}^{1} Z_{1}+F_{2}^{2} Z_{2},
\end{array} Z_{i}=T_{i}^{j} X_{j}, \quad Q_{1}=T_{1}^{j} B_{j}=\left(\begin{array}{ll}
0 & 0 \\
0 & 1
\end{array}\right) .\right.
$$

Решение первого из этих уравнений имеет вид $Z_{1}=Z_{10} \int Z_{10}^{-1} Q_{1} d t$, a $\dot{Z}_{10}=F_{1}^{1} Z_{10}$. Последнее уравнение эквивалентно матричному уравнению с постоянным коэффициентом и легко интегрируется:

$$
\begin{aligned}
\left(\dot{Z}_{10}=F_{1}^{1} Z_{10}\right) & \Longleftrightarrow\left(\frac{d Z_{10}}{d \tau_{1}}=\left(\begin{array}{ll}
0 & 1 \\
1 & 0
\end{array}\right) Z_{10}: \tau_{1}=\frac{1}{2} t^{2}\right) \\
& \Longleftrightarrow\left(Z_{10}=\exp \left(\begin{array}{ll}
0 & 1 \\
1 & 0
\end{array}\right) \tau_{1} C\right) .
\end{aligned}
$$

Полагая $C=E$, имеем

$$
Z_{10}=\exp \left(\begin{array}{ll}
0 & 1 \\
1 & 0
\end{array}\right) \tau_{1}=\left(\begin{array}{ll}
\operatorname{ch} \tau_{1} & \operatorname{sh} \tau_{1} \\
\operatorname{sh} \tau_{1} & \operatorname{ch} \tau_{1}
\end{array}\right)
$$

т.е.

$$
\begin{aligned}
Z_{1} & =\left(\begin{array}{ll}
\operatorname{ch} \tau_{1} & \operatorname{sh} \tau_{1} \\
\operatorname{sh} \tau_{1} & \operatorname{ch} \tau_{1}
\end{array}\right) \int\left(\begin{array}{cc}
\operatorname{ch} \tau_{1} & -\operatorname{sh} \tau_{1} \\
-\operatorname{sh} \tau_{1} & \operatorname{ch} \tau_{1}
\end{array}\right)\left(\begin{array}{ll}
0 & 0 \\
0 & 1
\end{array}\right) d t \\
& =\left(\begin{array}{ll}
0 & \operatorname{sh} \tau_{1} \int \operatorname{ch} \tau_{1} d t-\operatorname{ch} \tau_{1} \int \operatorname{sh} \tau_{1} d t \\
0 & \operatorname{ch} \tau_{1} \int \operatorname{ch} \tau_{1} d t-\operatorname{sh} \tau_{1} \int \operatorname{sh} \tau_{1} d t
\end{array}\right) .
\end{aligned}
$$

Зная $Z_{1}$, можно согласно (13) определить $Z_{2}=Z_{20} \int Z_{20}^{-1} F_{2}^{1} Z_{1} d t, \dot{Z}_{20}=F_{2}^{2} Z_{20}$. Так как $F_{2}^{2}$ имеет вид (11) при $\varphi_{21}=1, \varphi_{22}=-\varphi_{23}=t$, то $Z_{20}$ находится по формуле (14), в которой $\tau_{2}=0, a_{2}=\exp \left(t+\tau_{1}\right)-1, b_{2}=t$,

$$
Z_{20}=a_{2}\left(\begin{array}{cc}
1-t^{2} & t^{2} \\
-t^{2} & 1+t^{2}
\end{array}\right)
$$


Найдем подынтегральное выражение в $Z_{2}$. Имеем

$$
\begin{gathered}
Z_{20}^{-1} F_{2}^{1} Z_{1}=a_{2}^{-1}\left(\begin{array}{cc}
1+t^{2} & -t^{2} \\
t^{2} & 1-t^{2}
\end{array}\right)\left(\begin{array}{ll}
0 & 0 \\
0 & 1
\end{array}\right) Z_{1}=a_{2}^{-1}\left(\begin{array}{cc}
0 & -t^{2} \\
0 & 1-t^{2}
\end{array}\right) Z_{1} \\
=a_{2}^{-1}\left(\begin{array}{cc}
0 & -t^{2} R \\
0 & \left(1-t^{2}\right) R
\end{array}\right), \\
R=\operatorname{ch} \tau_{1} \int \operatorname{ch} \tau_{1} d t-\operatorname{sh} \tau_{1} \int \operatorname{sh} \tau_{1} d t .
\end{gathered}
$$

Следовательно,

$$
Z_{2}=a_{2}\left(\begin{array}{cc}
1-t^{2} & t^{2} \\
-t^{2} & 1+t^{2}
\end{array}\right)\left(\begin{array}{cc}
c_{1} & -\int a_{2}^{-1} t^{2} R d t \\
c_{2} & \int a_{2}^{-1}\left(1-t^{2}\right) R d t
\end{array}\right), \quad \dot{c}_{i}=0
$$

Из равенств

$$
\left(\begin{array}{cc}
0 & 1 \\
-1 & 0
\end{array}\right) X_{1}+\left(\begin{array}{cc}
0 & 1 \\
0 & 0
\end{array}\right) X_{2}=Z_{1}, \quad\left(\begin{array}{ll}
1 & 0 \\
0 & 0
\end{array}\right) X_{1}+\left(\begin{array}{ll}
1 & 0 \\
0 & 2
\end{array}\right) X_{2}=Z_{2}
$$

в соответствии с леммой 1 находим $X_{i}$. Так как из (3) имеем

$$
\begin{aligned}
\Delta(T) & =T_{1}^{1}\left(T_{2}^{2}-T_{2}^{1}\left(T_{1}^{1}\right)^{-1} T_{1}^{2}\right) \\
& =\left(\begin{array}{cc}
0 & 1 \\
-1 & 0
\end{array}\right)\left(\left(\begin{array}{ll}
1 & 0 \\
0 & 2
\end{array}\right)-\left(\begin{array}{ll}
1 & 0 \\
0 & 0
\end{array}\right)\left(\begin{array}{cc}
0 & -1 \\
1 & 0
\end{array}\right)\left(\begin{array}{ll}
0 & 1 \\
0 & 0
\end{array}\right)\right)=\left(\begin{array}{cc}
0 & 2 \\
-1 & 0
\end{array}\right),
\end{aligned}
$$

то $\delta(T) \neq 0$ и по формулам (6) находим

$$
X_{1}=\left(\begin{array}{cc}
0 & -1 \\
1 & 0
\end{array}\right) Z_{1}-\left(\begin{array}{cc}
0 & 0 \\
0 & \frac{1}{2}
\end{array}\right) Z_{2}, \quad X_{2}=\left(\begin{array}{cc}
0 & 1 \\
0 & 0
\end{array}\right) Z_{1}+\left(\begin{array}{cc}
1 & 0 \\
0 & \frac{1}{2}
\end{array}\right) Z_{2}
$$

Определим уравнения второго порядка, эквивалентные системе (21). Так как она является неоднородной, вместо (20) имеем $\ddot{X}_{i}=A_{i 2}^{j} X_{j}+\dot{B}_{i}+A_{i}^{j} B_{j}$. При этом $i$ и $j$ принимают лишь два значения, $j=(i \vee \bar{\imath})$, а $X_{\bar{\imath}}=\left(A_{i}^{\bar{\tau}}\right)^{-1}\left(\dot{X}_{i}-A_{i}^{i} X_{i}-B_{i}\right)$. Следовательно, $\ddot{X}_{i}=A_{i 2}^{i} X_{i}+A_{i 2}^{\bar{\tau}}\left(A_{i}^{\bar{\tau}}\right)^{-1} \times\left(\dot{X}_{i}-A_{i}^{i} X_{i}-B_{i}\right)+\dot{B}_{i}+A_{i}^{i} B_{i}$, что можно записать в виде

$$
\ddot{X}_{i}+P_{i} \dot{X}_{i}+Q_{i} X_{i}=R_{i}
$$

где

$$
\begin{gathered}
-P_{i}=A_{i 2}^{\bar{\tau}}\left(A_{i}^{\bar{\tau}}\right)^{-1}=\left(\dot{A}_{i}^{\bar{\tau}}+A_{i}^{j} A_{j}^{\bar{\tau}}\right)\left(A_{i}^{\bar{\tau}}\right)^{-1}, \\
-Q_{i}=P_{i} A_{i}^{i}+\dot{A}_{i}^{i}+A_{i}^{j} A_{j}^{i}, \quad R_{i}=\dot{B}_{i}+\left(A_{i}^{i}+P_{i}\right) B_{i}+A_{i}^{\bar{\tau}} B_{\bar{\imath}} .
\end{gathered}
$$


Таким образом, при $i=1(\bar{\imath}=2)$ имеем

$$
\begin{aligned}
& -P_{1}=\left(\left(\begin{array}{cc}
0 & -1 \\
\frac{1}{2} & -2
\end{array}\right)+\left(\begin{array}{cc}
0 & -t \\
\frac{1}{2}(1-t) & 0
\end{array}\right)\left(\begin{array}{cc}
0 & -t \\
\frac{1}{2} t & -1-t
\end{array}\right)\right. \\
& \left.+\left(\begin{array}{cc}
0 & -t \\
\frac{1}{2} t & 1+2 t
\end{array}\right)\right)\left(\begin{array}{cc}
0 & -t \\
\frac{1}{2} t & -1-2 t
\end{array}\right)^{-1} \\
& =\left(\begin{array}{cc}
0 \\
-t-\frac{1}{t}-\frac{1}{t}-\frac{3}{t^{2}} & 2 t+\frac{2}{t}
\end{array}\right) \text {, } \\
& -Q_{1}=\left(\begin{array}{cc}
\frac{1}{t} & 0 \\
t^{2}+\frac{1}{t}+\frac{1}{t^{2}}+\frac{3}{2} & -2 t-2-\frac{1}{t}
\end{array}\right)\left(\begin{array}{cc}
0 & -t \\
\frac{1}{2}(1-t) & 0
\end{array}\right) \\
& +\left(\begin{array}{cc}
0 & -1 \\
-\frac{1}{t} & 0
\end{array}\right)+\left(\begin{array}{cc}
0 & -t \\
\frac{1}{2}(1-t) & 0
\end{array}\right)+\left(\begin{array}{cc}
0 & -t \\
\frac{1}{2} t & -1-2 t
\end{array}\right)\left(\begin{array}{cc}
1 & t \\
-\frac{1}{2}(1+t) & 0
\end{array}\right) \\
& =\left(\begin{array}{cc}
t^{2} & -2 \\
2 t+\frac{1}{2}+\frac{1}{2 t} & -2 t-1-\frac{1}{t}
\end{array}\right) \text {, } \\
& R_{1}=\left(\left(\begin{array}{cc}
0 & -t \\
\frac{1}{2}(1-t) & 0
\end{array}\right)+\left(\begin{array}{cc}
-\frac{1}{t} & 0 \\
t+\frac{1}{t}+\frac{1}{t^{2}}+\frac{3}{2} & -2 t-2-\frac{1}{t}
\end{array}\right)\right)\left(\begin{array}{cc}
0 & -1 \\
0 & 0
\end{array}\right) \\
& =\left(\begin{array}{ll}
0 & \frac{1}{t} \\
0 & -\frac{1}{2} t-\frac{1}{t}^{\frac{1}{t^{2}}}-2
\end{array}\right) \text {. }
\end{aligned}
$$

Следовательно, найденное $X_{1}$ является решением уравнения

$$
\begin{aligned}
\ddot{X}_{1} & +\left(\begin{array}{cc}
-\frac{1}{t} & 0 \\
t+\frac{1}{t}+\frac{1}{t^{2}}+\frac{3}{2} & -2 t-2-\frac{1}{t}
\end{array}\right) \dot{X}_{1}+\left(\begin{array}{cc}
-t^{2} & 2 \\
-2 t-\frac{1}{2}-\frac{1}{2 t} & 2 t+1+\frac{1}{t}
\end{array}\right) X_{1} \\
& =\left(\begin{array}{cc}
0 & \frac{1}{t} \\
0 & -\frac{1}{2} t-\frac{1}{t}-\frac{1}{t^{2}}-2
\end{array}\right) .
\end{aligned}
$$

Аналогично, для $i=2$ имеем

$$
\begin{gathered}
-P_{2}=\left(\begin{array}{cc}
\frac{1}{t} & 2(t-1)+\frac{2}{t(t+1)} \\
\frac{1}{2} & 2(t+1)+\frac{1}{t+1}
\end{array}\right), \quad R_{2}=\left(\begin{array}{cc}
0 & -\frac{1}{t} \\
0 & 0
\end{array}\right), \\
-Q_{2}=\left(\begin{array}{cc}
t^{2}-t+1-\frac{1}{t(t+1)} & 2(2 t+1)\left(1-t-\frac{1}{t(t+1)}\right) \\
\frac{t}{2(t+1)}-1 & 2-(2 t+1)\left(1+\frac{1}{t+1}\right)
\end{array}\right),
\end{gathered}
$$

что определяет следующее уравнение второго порядка:

$$
\begin{aligned}
\ddot{X}_{2}- & \left(\begin{array}{cc}
\frac{1}{t} & 2(t-1)+\frac{2}{t(t+1)} \\
\frac{1}{2} & 2(t+1)+\frac{1}{t+1}
\end{array}\right) \dot{X}_{2}-\left(\begin{array}{cc}
t^{2}-t+1-\frac{1}{t(t+1)} & 2(2 t+1)\left(1-t-\frac{1}{t(t+1)}\right) \\
\frac{t}{2(t+1)}-1 & 2-(2 t+1)\left(1+\frac{1}{t+1}\right)
\end{array}\right) X_{2} \\
& =\left(\begin{array}{cc}
0 & -\frac{1}{t} \\
0 & 0
\end{array}\right) .
\end{aligned}
$$

В заключение отметим, что все полученные результаты полностью переносятся на правосторонние СМЛОДУ.1, разумеется, при коммутации матричных сомножителей во 
всех произведениях. Их можно использовать и для определения условий разрешимости в квадратурах линейных систем с разносторонним умножением

$$
\dot{X}_{i}=A_{i}^{j} X_{j}+X_{k} B_{i}^{k}+C_{i}
$$

которые введением новых переменных сводятся к решению отдельных лево- и правосторонних систем.

Более того, путем редуцирования матричных уравнений [8] предлагаемьй метод интегрирования систем может быть распространен и на СМЛОДУ.1 с двусторонним умножением

$$
\dot{X}_{i}=\sum_{k=1}^{K} \sum_{j=1}^{N} A_{i k}^{j} X_{j} B_{i k}^{j}+C_{i}
$$

позволяя существенно обобщить теорему 2 этой работы.

\section{СПИСОК ЦИТИРОВАННОЙ ЛИТЕРАТУРЫ}

[1] Адо И. Д. Представление алгебр Ли матрицами // УМН. 1947. Т. 11. №6 (22). С. 159-173.

[2] Понтрягин Л. С. Обыкновенные дифференциальные уравнения. М.: Наука, 1970.

[3] Гантмахер Ф.Р. Теория матриц. М.: Наука, 1967.

[4] Фаддеев Д.К., Фаддеева В.Н. Вычислительные методы линейной алгебры. М.-Л.: ГИФМЛ, 1963.

[5] Деревенский В. П. Экспоненциальное решение матричных линейных дифференциальных уравнений первого порядка // Изв. вузов. Матем. 1981. № 7. С. 31-33.

[6] Wei J., Norman E. On global representations of the solutions of linear differential equations // Proc. Amer. Math. Soc. 1964. V. 15. № 2. P. 327-334.

[7] Деревенский В. П. Интегрируемость уравнения Риккати и линейного однородного дифференциального уравнений второго порядка // Изв. вузов. Матем. 1987. № 5. С. 33-40.

[8] Деревенский В.П. Матричные двусторонние линейные дифференциальные уравнения // Матем. заметки. 1994. Т. 55. №1. С. 35-42. 